\title{
Constructing Ultralong Near-infrared Organic Phosphorescence Materials with 732 nm through One-axis Two-wing Guest-Host Strategy for Bioimaging
}

Fuming Xiaoł $\ddagger^{a}$, Heqi Gaot $\ddagger^{b}$, Yunxiang Lei ${ }^{* a}$, Miaochang Liu ${ }^{a}$, Xiaoyan Zheng ${ }^{c}$, Zhengxu $\mathrm{Cai}^{c}$, Xiaobo Huang ${ }^{* a}$, Huayue $\mathrm{Wu}^{a}$ and Dan Ding ${ }^{* b}$

${ }^{a}$ School of Chemistry and Materials Engineering, Wenzhou University, Wenzhou 325035, P. R. China

${ }^{\mathrm{b}}$ Collaborative Innovation Center of Chemical Science and Engineering, Nankai University, Tianjin 300071, P. R. China

${ }^{\mathrm{c}}$ School of Materials Science \& Engineering, Beijing Institute of Technology, Beijing 10081, P. R. China

E-mail: yunxianglei@wzu.edu.cn (Y. Lei); xiaobhuang@wzu.edu.cn (X. Huang); dingd@ nankai.edu.cn (D. Ding)

$\$$ These authors contributed equally.

\begin{abstract}
Organic near-infrared room temperature phosphorescence (RTP) materials have unparalleled advantages in bioimaging due to their better penetrability. However, limited by the energy gap law, organic matters with long wavelengths (> $700 \mathrm{~nm})$ and long lifetimes (> $100 \mathrm{~ms}$ ) have not been reported so far. In this work, we have obtained organic RTP materials with long wavelengths $(657-732 \mathrm{~nm}$ ) and long lifetimes (102-324 ms) for the first time through the one-axis two-wing guest-host strategy. The one axis refers to that the guest molecule has sufficient conjugation to reduce the lowest triplet energy level and the two wings refer to that the host assists the guest in exciton transfer and inhibits the non-radiative transition of guest excitons. These materials exhibit good tissue penetration in bioimaging. Thanks to the characteristic of long lifetime and long wavelength emissive RTP materials, the tumor imaging in living mice with a signal to background ratio value as high as 43 is successfully realized. This work provides a practical solution for the construction of organic RTP materials with both long wavelengths and long lifetimes used in bioimaging.
\end{abstract}

\section{Introduction}

Organic room temperature phosphorescence (RTP) materials with persistent emission can effectively avoid the interference of environmental self-luminescence, and the organic matters often have the advantages of low toxicity and good biocompatibility. ${ }^{1-10}$ Therefore, constructing organic RTP materials is considered to be an ideal means for tissue imaging, tumor diagnosis, and drug tracking. ${ }^{10-15}$ But so far most phosphorescence materials have poor biological tissue permeability due to their 
short emission wavelength (less than $580 \mathrm{~nm}$ ), ${ }^{16-27}$ which makes the phosphors have better imaging only in the shallow part of organism, so it is urgent to construct near-infrared phosphorescent materials. Although researchers have developed some red RTP materials, such as boron fluoride, carbazole, and naphthalene diimides, those with wavelengths exceeding $650 \mathrm{~nm}$ are very rare. ${ }^{28-33}$ Moreover, presumably limited by the energy gap law, these red RTP materials have short phosphorescence lifetimes which are not conducive to bioimaging (Supplementary Scheme S1).

The establishment of ultralong RTP materials with long emission wavelength inevitably means the reduction of the lowest triplet $\left(T_{1}\right)$ level of the materials, but the lower $\mathrm{T}_{1}$ level will bring two major obstacles to the phosphorescence emission. The first obstacle is that the lower $T_{1}$ increases the band gaps between $S_{1}$ and $T_{1}\left(\Delta E_{\mathrm{ST}}\right)$, which is not conducive to the intersystem crossing (ISC) of excitons. Another obstacle is that the lower $T_{1}$ level easily causes excitons to be consumed in a non-radiative way, resulting in a significant reduction in the lifetime and intensity of phosphorescence emission (Scheme 1a). Therefore, under the premise of keeping materials with a low $\mathrm{T}_{1}$, improving the ISC ability of excitons and suppressing the non-radiative transitions of excitons are the keys to achieving ultralong near-infrared phosphorescence. Recently, guest-host materials have gradually attracted more attention, ${ }^{34-47}$ because the host matrix can inhibit the non-radiative transition of the guest excitons in the guest-host system. ${ }^{42-47}$ Additionally, some research results have shown that there is energy synergy between the host and guest molecules, which can effectively assist the guest molecules to transfer the excited state energy. ${ }^{39-43}$ Therefore, the guest-host system provides a new strategy for the construction of organic RTP materials with long wavelength and long lifetime.

On this basis, we try to construct ultralong near-infrared RTP materials through one-axis two-wing guest-host strategy in the first time. In this work, the guest pyrene derivatives with high conjugation are regarded as the central axis, their high conjugation can reduce the $T_{1}$ levels of the molecules, then ensuring that the materials have long phosphorescence wavelengths. The benzophenone (BPO) molecule is chosen as the host molecule, which can act as two wings. One wing refers to that the host matrix can assist the transfer of guest excitons and the other wing refers to that the host matrix can restrict the motions of guest molecules, thereby suppressing the non-radiative transition of guest excitons (Scheme 1c). The results show that the designed guest-host materials successfully have strong red afterglow visible to the naked eye. With the increase of conjugation degree of guest molecules, the phosphorescence wavelengths of the guest-host materials are red-shifted from $657 \mathrm{~nm}$ to $732 \mathrm{~nm}$. More importantly, our newly developed guest-host materials have a long phosphorescence lifetime of 102-324 ms. To the best of our knowledge, this is the 
first RTP material with both long wavelength (> $700 \mathrm{~nm}$ ) and long lifetime (> $100 \mathrm{~ms})$. The comparative experiments of the molten state and the crystalline state prove that it is necessary for materials to possess RTP properties that the host restricts the motion of guest molecules. The molecular dynamics (MD) simulation further shows that there is a strong interaction between the host and the guest, which can suppress the non-radiative transition of the guest excitons. Moreover, the experimental results also confirm that the host molecules show a synergistic effect to the guest at excited state. As a proof-of-concept, the materials have been used for precise lymph node mapping and armpit tumor labeling with a high SBR of 55 and 43, respectively. The long wavelength helps to reduce the tissue scattering and long lifetime further avoids the interference of autofluorescence in bioimaging. Thus, the phosphorescence materials with both kinds of properties can provide more unambiguous tumor imaging.

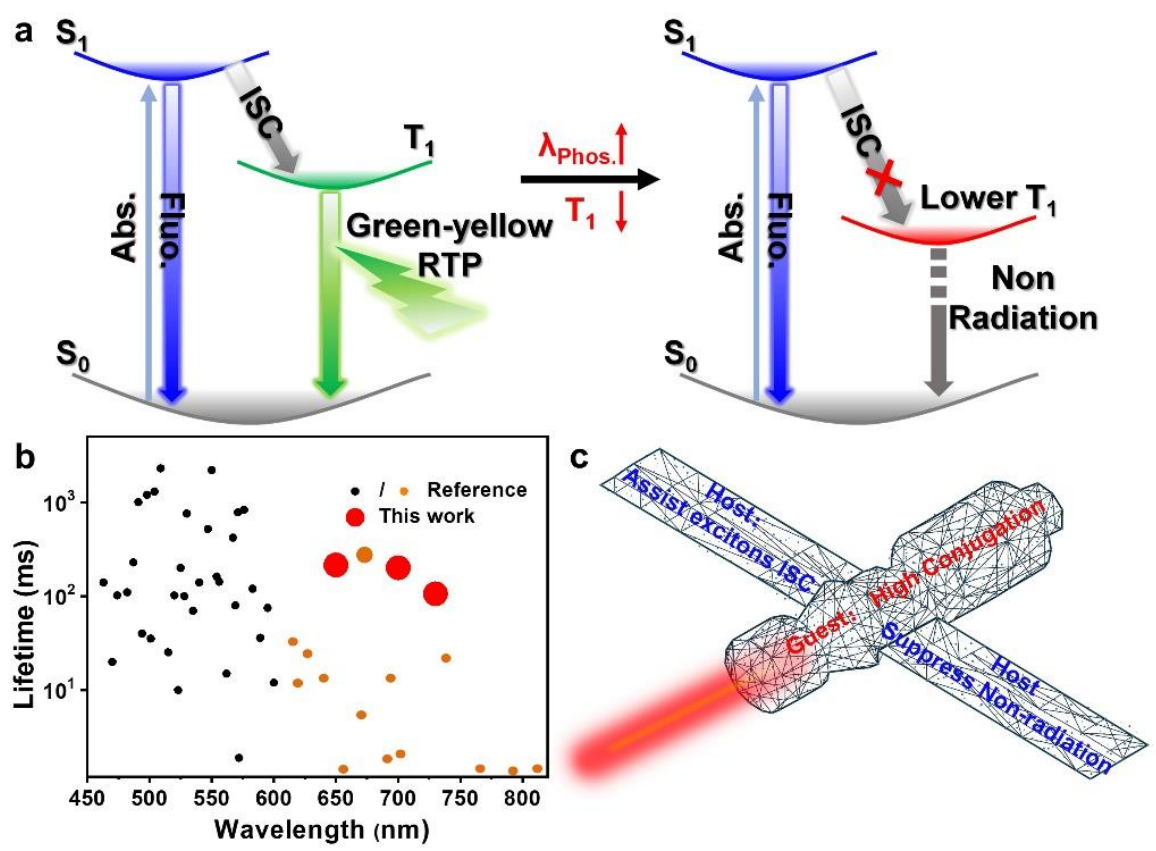

Scheme 1. | Design concept of the guest/host system. a, Phosphorescence performance distribution of RTP materials. b, Problems in constructing ultralong near-infrared RTP materials. $\mathbf{c}$, Strategy for constructing ultralong near-infrared RTP materials in this work.

\section{Results and Discussion}

The guest molecules are based on the pyrene unit, in which introducing anisole or $\mathrm{N}, \mathrm{N}$-dimethylaniline groups on one or both sides of pyrene through Suzuki reaction is to increase the molecular conjugation (Fig. 1a, Supplementary Scheme S2). The five guests show good solubility in chloroform, tetrahydrofuran, and dimethyl sulfoxide. The molecular structures and purities of the target compounds were confirmed by NMR spectroscopy, single-crystal X-ray diffraction, and high performance liquid Chromatography (Fig. S1). As the conjugation of the guests increases, the maximum absorption peaks are red-shifted from $343 \mathrm{~nm}$ to $378 \mathrm{~nm}$ (Fig. S2a), and the 
corresponding fluorescence peaks are also red-shifted from $381 \mathrm{~nm}$ to $467 \mathrm{~nm}$ (Fig. $\mathrm{S} 2 \mathrm{~b})$. The host BPO is directly purchased commercially without further processing. BPO has low melting points $\left(48^{\circ} \mathrm{C}\right)$ and stable subcooling states and thus the guests dispersed in the host can be fabricated by the melt-casting method. Because the concentration of the guests is very important in determining the RTP properties of the guest-host materials, we first prepared a series of Py/BPO guest-host materials with different guest-host molar ratios (1:50-1:50000) to optimize the luminescence performance. The strongest phosphorescence intensity was obtained with the guest-host molar ratio of 1:1000 (Fig. S4), which is in line with our previous works. $^{40-43}$ Four other guest-host materials (MOPy/BPO, MAPy/BPO, DMOPy /BPO, and DMAPy/BPO, Fig. 1a) with the guest-host molar ratio of 1:1000 were further prepared, and the luminescence characteristics of the host-guest systems were systematically investigated.

Five guest-host materials show blue to cyan fluorescence emission under the excitation source (Fig. 1b) and the maximum emission peaks of the guest-host materials are red-shifted from $416 \mathrm{~nm}$ to $483 \mathrm{~nm}$ as the conjugation of the guest increases (Table 1, Supplementary Fig. S5). Importantly, after removing the irradiation, except for DMAPy/BPO, the other four guest-host materials have a deep red afterglow visible to the naked eye for about $3 \mathrm{~s}$, revealing RTP properties (Fig. 1b). Delayed spectra further show that the guest-host materials have two phosphorescence peaks, which are the fine structure caused by energy level vibration. Similarly, with the increase of guest conjugation, the phosphorescence peaks of the guest-host materials are red-shifted from $657 \mathrm{~nm}$ to 732 or $600 \mathrm{~nm}$ to $681 \mathrm{~nm}$ (Table 1, Fig. 1c). This indicates that the guest-host materials have achieved deep red or even near-infrared phosphorescence emission, which is a group of guest-host materials with the longest phosphorescence wavelengths so far. The Commission Internationale de l'Eclairage (CIE) coordinates further indicate that the guest-host materials have a very deep phosphorescent color $(0.63,0.35 ; 0.64,0.34 ; 0.65,0.33 ; 0.69,0.30 ; 0.70$, 0.29) (Fig. S6). It is worth mentioning that, unlike most red RTP materials which have short phosphorescence lifetimes, the phosphorescence lifetimes of this guest-host system are 102-324 ms (Table 1. Fig. 1d). Moreover, the guest-host materials have satisfactory luminous intensities and the phosphorescence quantum efficiency is in the range of $4.2 \%-9.2 \%$ (Table 1 ). The above results fully prove that we have successfully developed a group of ultralong near-infrared RTP materials through the one-axis two-wing guest-host strategy. 

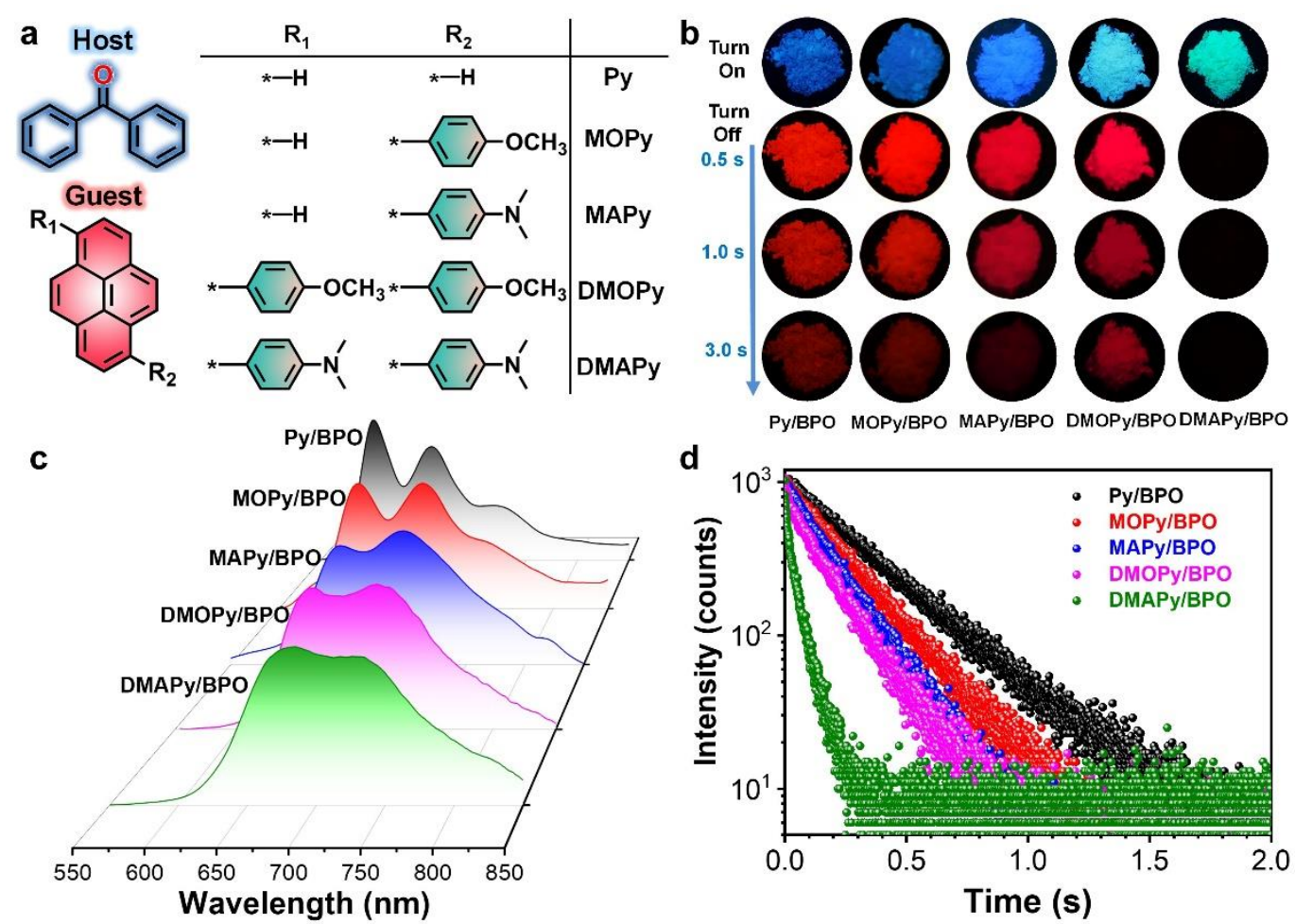

Fig. 1. | Photoluminescence properties of the guest/host system. a, The molecular structures of guest and host molecules. b. The fluorescence (top) and phosphorescence (down) images of the guest-host materials. c, Phosphorescence spectra of host-guest materials. Excitation wavelength: $380 \mathrm{~nm}$ d, Phosphorescence decay curves of host-guest materials. Excitation wavelength: $380 \mathrm{~nm}$.

Table 1. Photophysical data of the guest-host materials

\begin{tabular}{lcccccc}
\hline Sample & \multicolumn{3}{c}{ Fluo. } & \multicolumn{3}{c}{ Phos. } \\
& $\lambda_{\text {em }}$ & $\begin{array}{c}\boldsymbol{\Phi}_{\mathbf{F}} \\
(\mathbf{n m})\end{array}$ & $\begin{array}{c}\boldsymbol{\tau} \\
(\mathbf{n s})\end{array}$ & $\begin{array}{c}\boldsymbol{\lambda}_{\mathrm{em}} \\
(\mathbf{n m})\end{array}$ & $\begin{array}{c}\boldsymbol{\Phi}_{\mathbf{P}} \\
(\boldsymbol{\%})\end{array}$ & $\begin{array}{c}\boldsymbol{\tau} \\
(\mathbf{m s})\end{array}$ \\
\hline Py/BPO & 415 & 14.2 & 1.43 & 600,657 & 9.2 & 327,324 \\
MOPy/BPO & 424 & 13.4 & 2.03 & 623,680 & 8.0 & 215,210 \\
MAPy/BPO & 440 & 15.2 & 1.98 & 643,697 & 6.3 & 201,198 \\
DMOPy/BPO & 471 & 12.3 & 2.19 & 657,713 & 5.4 & 180,175 \\
DMAPy/BPO & 483 & 16.1 & 2.32 & 681,732 & 4.2 & 106,102 \\
\hline
\end{tabular}

Generally, the triplet excitons are unstable and easily consumed by the motion of molecules, leading to the quenching of phosphorescence. But for the guest-host system, the host molecules can provide a rigid environment to restrict the motion of the guest molecules, thus making the guest emit phosphorescence. ${ }^{43,44}$ We first collected the phosphorescence emissions of the guest molecules at low temperature $(77 \mathrm{~K})$ to verify whether the phosphorescence wavelengths of this guest-host system are emitted by the guest molecules. The phosphorescence spectra show that the guests also have two fine peaks at $77 \mathrm{~K}$, and the emission peaks are also red-shifted from 
$596 \mathrm{~nm}$ to $665 \mathrm{~nm}$ or $652 \mathrm{~nm}$ to $725 \mathrm{~nm}$ (Fig. Supplementary S9), which are almost completely consistent with the phosphorescence wavelengths of the corresponding guest-host materials. The results confirm that the phosphorescence in the guest-host system is emitted by the guest molecules. Taking advantage of the low melting point of host, the influence of the host morphology on the phosphorescence performance of the guest-host system was discussed. As shown in Fig. 2a and Fig. 2b, the Py/BPO molten state at room temperature (subcooling state) show only fluorescence but no phosphorescence. However, when the guest-host material begins to crystallize, the material shows bright red phosphorescence. This clearly proves that the restriction of the host matrix on the guest molecular motion is a necessary factor for the guest host-system to have RTP properties.

The concrete microenvironment of molecules, such as molecular configuration, intermolecular distance, and intermolecular interaction plays an important role in determining the photophysical phenomena of materials. But it is difficult to get the co-crystal of host-guest because of very trace amount of guest molecules $(<0.1 \%)$ in the entire materials. Moreover, the traditional characterization methods, such as X-ray diffractometer, scanning electron microscope (SEM), and transmission electron microscope (TEM) are difficult to accurately investigate the concrete molecular conformation of guest in the host matrix. Therefore, we simulated the molecular conformations of Py molecules in the BPO matrix using MD simulations. ${ }^{41}$ The initial Py/BPO model was based on BPO crystal. As shown in Fig. 2c, a BPO molecule is removed from the BPO crystal and the vacancy is filled with by a $\mathbf{P y}$ molecule. The obtained Py/BPO model possesses 1:191 molar ratio of Py to BPO. Starting from the initial Py/BPO configuration, we perform further 10 ns production MD simulations to relax the whole guest-host system using GROMACS software package (version 5.1.5, details in SI). Compared with the conformations of BPO molecules in single crystal, the corresponding conformations of the BPO molecules adjacent to the guest Py molecule in Py/BPO guest-host system is slightly different because the twisted angles increases slightly after doping (Fig. S10). This is because the spatial volume of Py molecule is larger than that of BPO molecule. However, due to the very small proportion of the guest $\mathbf{P y}$ in the guest-host system, it has minimal impact on the overall arrangement of the host matrix. Therefore, the stacking of BPO molecules in the simulated guest-host system is almost comparable to that of the single crystal (Supplementary Fig. S11, S12). The XRD results also confirm that there is almost no change in the arrangement of the BPO host before and after doping with guest molecules (Supplementary Fig. S13). Therefore, it is reasonable and feasible to simulate the microenvironment of the guest-host system by MD simulations. According to the simulated Py/BPO model, we first analyze the relative spatial 
positions of the Py molecule in the BPO matrix, as shown in Fig. 2d, the distances between the guest molecule and the host molecules in six directions (up, down, front, back, left, and right) are $2.3 \AA-3.1 \AA$. It clearly shows that the guest molecules are in a relatively dense matrix environment, which can effectively inhibit the motion of the guest molecules. More importantly, although the distances between the host and the guest are relatively close, the host has a twisted molecular conformation, so there is no $\pi---\pi$ interaction between the host and the guest which is not conducive to luminescence. On the contrary, multiple $\mathrm{C}-\mathrm{H}---\pi$ interactions between the host molecules and Py molecule are observed with close distances (2.3 $\mathrm{A}-3.2 \AA$, red line) (Fig. 2e), and the average distance between Py molecule and surrounding host molecules is only $2.77 \AA$. In addition, the C-H---O interactions between the Py molecule and host molecules are also observed with close distances $(2.5 \AA$ and $2.6 \AA$, blue line) (Fig. 2e). The above analysis shows that the host matrix in the guest-host system provides a relatively closer and stronger interaction to the guest molecules, which can effectively restrict the non-radiative decay channel after the Py molecule is doping.

(a)
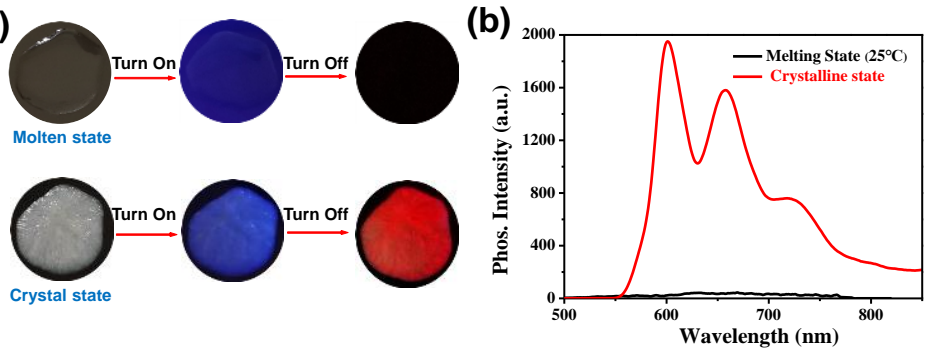

(d)

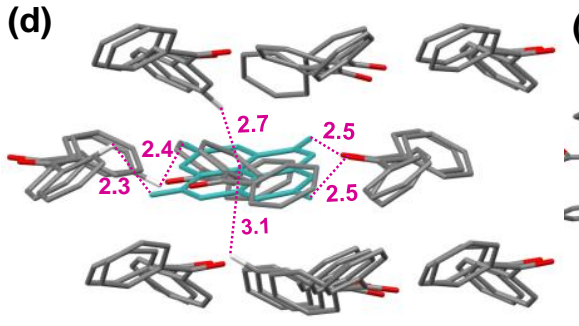

(e)

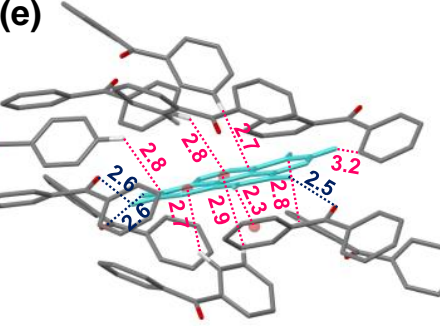

(c)

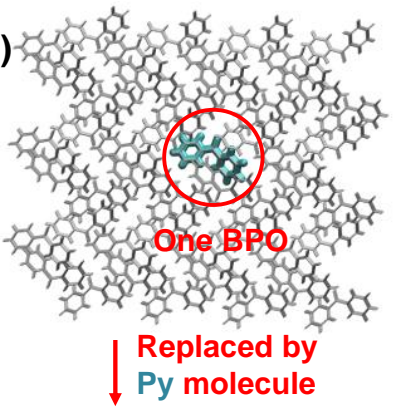

Fig. 2. Non-radiative suppression of guest excitons by host matrix. a, Photographs of $\mathbf{P y} / \mathbf{B P O}$ in different states. b, Phosphorescence spectra of Py/BPO. c, Model setup of Py/BPO guest-host system. d, The spatial distances between the Py molecule and the surrounding BPO molecules. e, The interaction distances of C-H--- $\pi$ or C-H---O interactions between $\mathbf{P y}$ molecule and surrounding BPO molecules. The distances between each phenyl ring center of Py molecule and the hydrogen atom of the BPO molecules are labelled by red line. The corresponding distances between the oxygen atom of BPO and the hydrogen atoms of Py are labelled by blue line. 
The rigid environment provided by the matrix is a necessary factor for the guest-host system to have RTP characteristics. However, whether the host molecules only play a role of physical restriction in guest-host system? We choose sulfonyldibenzene (SOB), sulfinyldibenzene (SIB), or diphenylphosphine oxide (PPO) which also has good crystallinity and similar structure to BPO as the host (Fig. 3a), and the MAPy as the guest. Three guest-host materials (MAPy/SOB, MAPy/SIB, and MAPy/PPO) with guest-host molar ratio of 1:1000 were prepared. Unfortunately, although the three guest-host materials have strong cyan fluorescence under the UV lamp, there is no red afterglow after the UV lamp is removed (Fig. 3b). The fluorescence spectra show that the fluorescence wavelengths of the three guest-host materials are around $430 \mathrm{~nm}$ (Fig. S14a), and the fluorescence quantum yields of the three guest-host materials are as high as $63 \%, 71 \%$ and $76 \%$, respectively. Such high luminous intensity proves that the host indeed inhibits the motion of the guest molecules. However, the delayed spectra display that the guest-host materials have almost no phosphorescence emission (Fig. S14b). It is worth mentioning that although the phosphorescence of MAPy/SOB and MAPy/SIB guest-host materials are very weak, there is an emission peak near $670 \mathrm{~nm}$, which once again proves that the phosphorescence in the guest-host material is emitted by the guest molecules. The above comparative experiments show that the rigid environment provided by the host to restrict the motion of guest molecules is only a necessary factor, not a sufficient factor, for the guest host materials to have RTP properties.

(a)
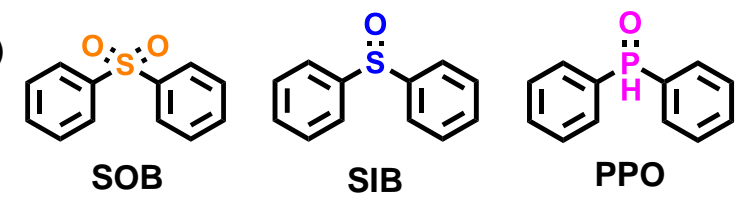

(b)

SOB

(b)
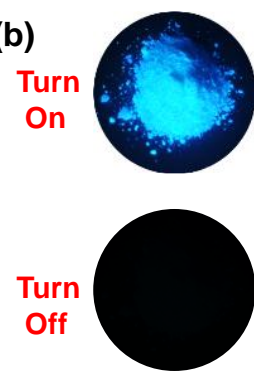

MAPy/SOB
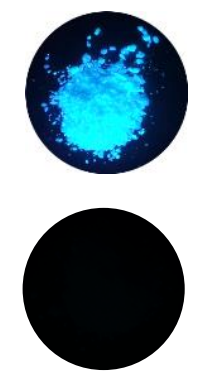

MAPy/SIB

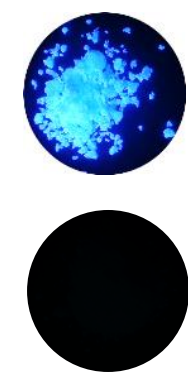

MAPy/PPO

Fig. 3. | Luminescence properties of guest molecules in other hosts. a, Molecular structure of the reference hosts. $\mathbf{b}$, Luminescent images of the reference guest-host materials.

Researchers have gradually discovered that the energy transfers between the host and guest molecules play a vital role in the phosphorescence activity. Among them, the Förster resonance energy transfer (FRET) is considered to be the reason why some 
guest-host materials have RTP properties. ${ }^{24,39}$ In this work, to verify whether there is FRET between the host and the guest, the absorption and excitation spectra of host BPO and guest MAPy were investigated. As shown in Fig. 4a, the absorption and excitation wavelengths of host BPO only reach $418 \mathrm{~nm}$, whereas the absorption and excitation wavelengths of guest MAPy reach $465 \mathrm{~nm}$. Therefore, we first investigated the phosphorescence emission of MAPy/BPO material at different excitation wavelengths. The results show that even if the excitation wavelength is extended to $440 \mathrm{~nm}$, the MAPy/BPO powder maintains a strong phosphorescence emission (Fig. 4b) and has a red afterglow visible to the naked eye after removing the $420 \mathrm{~nm} \mathrm{UV}$ lamp (Fig. 4c). These results clearly demonstrate that the phosphorescence of MAPy/BPO does not come from the energy absorbed by the host matrix, but from the energy absorbed by the guest molecules. Therefore, the FRET between the host and the guest is ruled out. In our previous works, we found that the host could assist the excitons of the guests in energy transfer. ${ }^{41-43}$ So in this work, we first tested the excitation spectra (fluorescence emission/420 nm) of guest MAPy in common solvents (toluene, THF, and N,N-dimethylformamide/DMF) and host BPO (molten state and crystal state). As show in Fig. 4d, the results show that the maximum excitation wavelengths of MAPy in common solvents are $346 \mathrm{~nm}$, while the maximum excitation wavelengths in the molten and crystal host are red-shifted to 392 $\mathrm{nm}$ and $387 \mathrm{~nm}$, respectively. And the excitation spectra of phosphorescence emission $(660 \mathrm{~nm})$ also show that the maximum excitation wavelengths of MAPy in common solvents are significantly longer than that of the crystal host (Fig. 4e). Hence, it can be judged that the host not only acts as the rigid matrix, but also changes the transfer process of the guest energy in the excited state. Furthermore, the phosphorescence lifetimes of guest molecules at $77 \mathrm{~K}$ only are $12 \mathrm{~ms}-23 \mathrm{~ms}$ (Fig. 4f), which are much shorter than that of the host matrix. This also shows that the host matrix prolongs the intersystem crossing process of the guest excitons. Based on the above experimental results and our previous works, ${ }^{41-43}$ we think that the $\mathrm{T}_{1}$ of the host can be the bridge between the $S_{1}$ and $T_{1}$ of the guest (Fig. $4 \mathrm{~g}$ ), so the excited energy from guest can transfer from the $S_{1}$ to $T_{1}$ of the guest through the path of the $T_{1}$ of the host. In order to verify the rationality of the mechanism, density functional theory calculations were carried out to obtain the singlet and triplet energy levels of the host and guest molecules. The lowest singlet state $\left(\mathrm{S}_{1}\right)$ and lowest triplet state $\left(\mathrm{T}_{1}\right)$ of the five guest molecules are 3.01-3.49 eV and 1.84-2.15 eV, respectively (Fig. 4h). The $\Delta \mathrm{E}_{\mathrm{ST}}$ of the guest molecules are in the range of 1.17-1.34 eV and such large energy gaps make it difficult for ISC of excitons. However, the band gaps between the $\mathrm{S}_{1}$ state of the guests and the $\mathrm{T}_{1}$ state of the host are only $0.11-0.59 \mathrm{eV}$ (Fig. 4h), which is very beneficial for intersystem crossing of excitons. Therefore, the synergy action of 
host-guest is also an important factor for the phosphorescent activity of the guest-host materials.
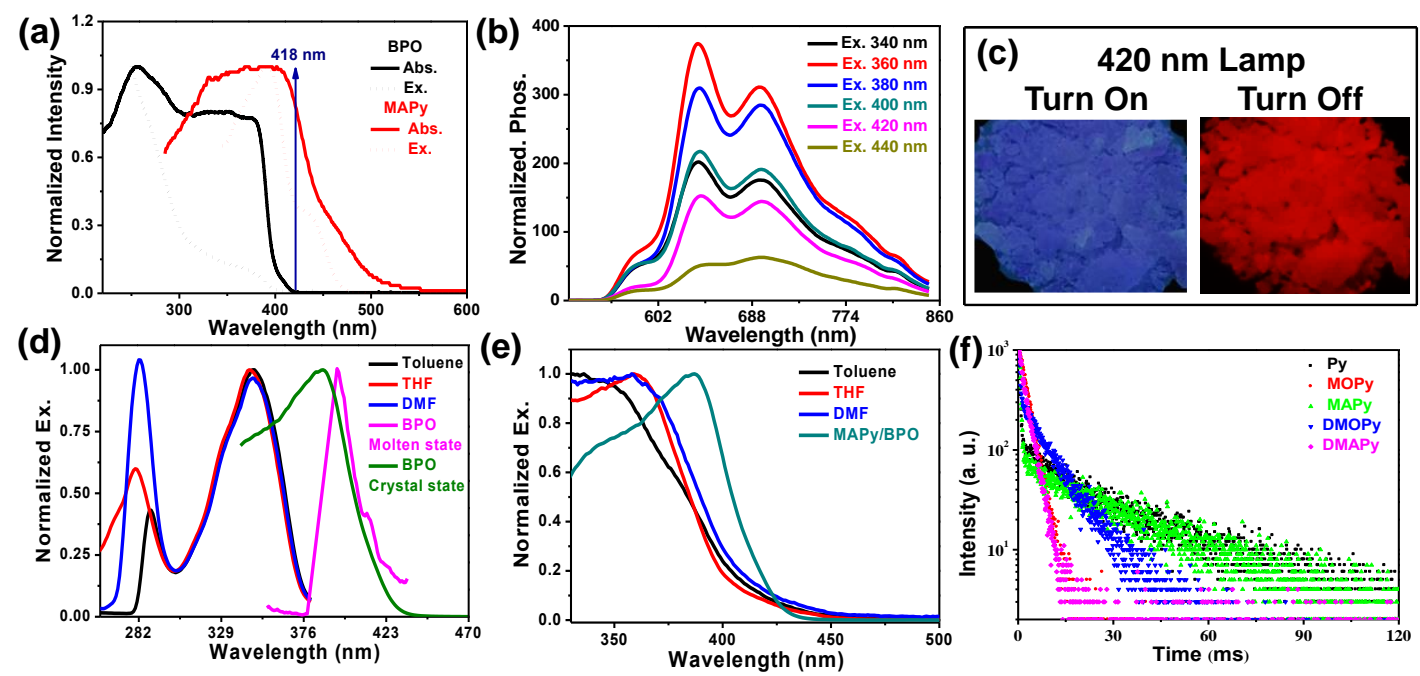

(g)
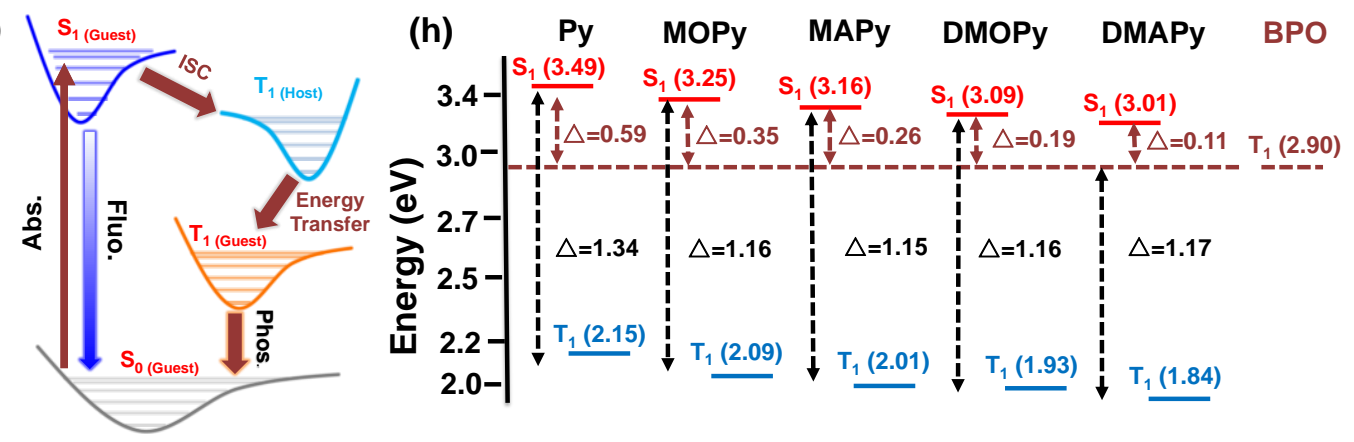

Fig. 4. | Energy transfer between guest and host. a, Excitation spectra of host BPO powder and guest MAPy powder. b, Phosphorescence spectra of guest-host material MAPy/BPO at different excitation wavelengths. c, Luminescence photos of the MAPy/BPO powder. d, e, Excitation spectra (fluorescence)/(phosphorescence) and of MAPy in different solvent and molten state host (Concentration: $1 \times 10^{-4} \mathrm{~mol} / \mathrm{L}$ ). f, Phosphorescence decay curves of the guests in $77 \mathrm{~K}$ (Ex: 380 $\mathrm{nm}$; Concentration: $1 \times 10^{-4} \mathrm{~mol} / \mathrm{L}$; Solvent: 2-methyltetrahydrofuran). g, Proposed transfer path between guest and host. $\mathbf{h}$, The energy levels of BPO and five guests.

It is well known that long wavelength emission is beneficial to reduce tissue scattering and enhance tissue penetration, which can improve bio-imaging quality. Encouraged by the excellent properties of these near-infrared RTP materials, the application in bioimaging was investigated next. Encouraged by the excellent properties of these near-infrared RTP materials, the application in bioimaging was investigated next. Because DMAPy/BPO exhibits the longest wavelength with a quite long lifetime among these materials, DMAPy/BPO and the biocompatible amphiphilic copolymer PEG-b-PPG-b-PEG (F127) were selected as the core of nanoparticles and the encapsulation matrix, respectively. To ensure our DMAPy/BPO NPs accessible in vivo with good RTP performance, a top-down method was employed to formulate nanoparticles (NPs). ${ }^{[48,49]}$ In order to verify the advantages of 
long wavelength RTP materials, the short wavelength RTP material DOB/BPO reported in our previous work was selected as the control and DOB/BPO NPs were prepared by the same method. ${ }^{[50]}$ Dynamic light scattering (DLS) and transmission electron microscopy (TEM) data displayed that both DMAPy/BPO and DOB/BPO nanoparticles hold an near-spherical morphology with a mean hydrodynamic diameter of $\approx 100 \mathrm{~nm}$ (Fig. 5a and Supplementary Fig. S15). Both kinds of nanoparticles revealed strong resistance to photobleaching, indicative of little change in their intensities after eight cycles of stimulation or eighty minutes of $365 \mathrm{~nm}$ UV light irradiation. (Fig. 5b, Supplementary Fig. S16 and Fig. S17) We further verified the promise for the quantitative conversion of phosphorescence intensity into NPs concentration. The phosphorescence intensities of DMAPy/BPO NPs and DOB/BPO NPs were captured at $t=10 \mathrm{~s}$ post-excitation, which possess a good linear relationship with varying concentrations of nanoparticles (Fig. 5c, Supplementary Fig. S18). As displayed in Fig. 5d, DMAPy/BPO NPs and DOB/BPO NPs showed the main phosphorescence signals under Dsred $(575-650 \mathrm{~nm})$ filter and GFP $(515-575 \mathrm{~nm})$ filter, respectively. This result is consistent with their phosphorescence spectra. As the tissue penetration is a considerable challenge of bioimaging in vivo, the tissue penetration depths of the nanoparticles were compared between DMAPy/BPO NPs and DOB/BPO NPs. As shown in Fig. 5e and Fig. 5f, the phosphorescence signals of both DMAPy/BPO NPs and DOB/BPO NPs were decreased with increased thickness of chicken breast tissue. The ultra-high signal to background ratio (SBR) signals derived from the advantages of RTP materials can be observed without chicken tissue covered. However, the inherent limit of short wavelength leads to relatively low tissue penetration (SBR=5.4 at the thickness of $7.5 \mathrm{~mm}$ ). In contrast, the NIR phosphorescence signal of the DMAPy/BPO NPs can still be detected (SBR=15) under a $12.5 \mathrm{~mm}$ thickness coverage of chicken tissue. This result revealed that the excellent capability of deep tissue imaging due to the NIR emission of the nanoparticles and without excitation. After we verified that both kinds of nanoparticles had good cytocompatibility (Fig. 5g), we further investigated phosphorescent performance of DMAPy/BPO NPs in varying metal ions (widespread in vivo) and tissue homogenates to confirm the bioimaging feasibility in vivo. Upon irradiation for $1 \mathrm{~min}$ by a $365 \mathrm{~nm}$ handheld UV lamp, the phosphorescence signals of DMAPy/BPO NPs incubated with varying metal ions were collected by IVIS bioimaging instrument under the same conditions, immediately. However, it is found that the ferric ions (including $\mathrm{Fe}^{2+}$ and $\mathrm{Fe}^{3+}$ ) can greatly quenched the phosphorescence signals compared to the signals in $\mathrm{Na}^{+}$(Fig. 5h). And this result might be attributed to the interaction between ferric ions with outer vacant orbital and O/N heteroatoms with lone pair electrons in DMAPy/BPO ${ }^{[51]}$ Further, it is found that 
the DMAPy/BPO NPs exhibit different phosphorescence quench behaviors in different tissue homogenates and blood (Fig. 5i). Compared with the phosphorescence signal in PBS, the signals of DMAPy/BPO NPs were greatly quenched in the blood and blood-rich-tissues (such as heart, liver etc.) due to the quenching effect of $\mathrm{Fe}^{2+}$ and $\mathrm{Fe}^{3+}$ ions, which would be beneficial for the imaging of tumors.
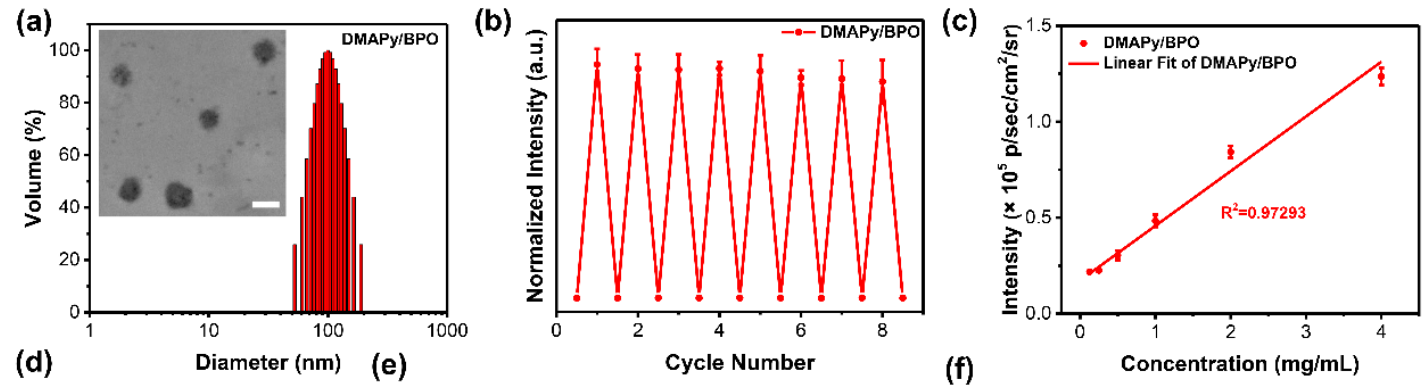

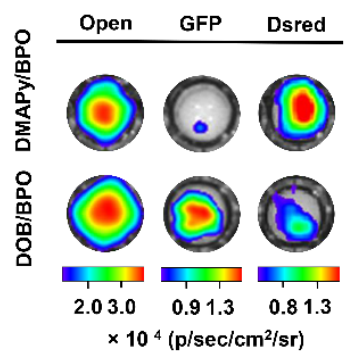

(g)

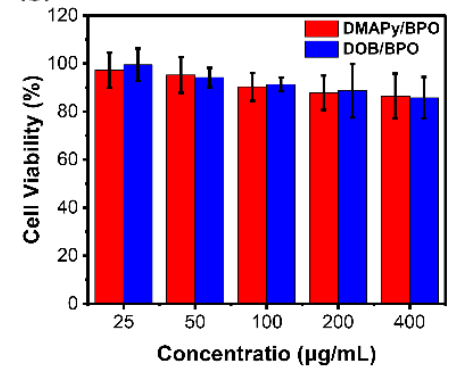

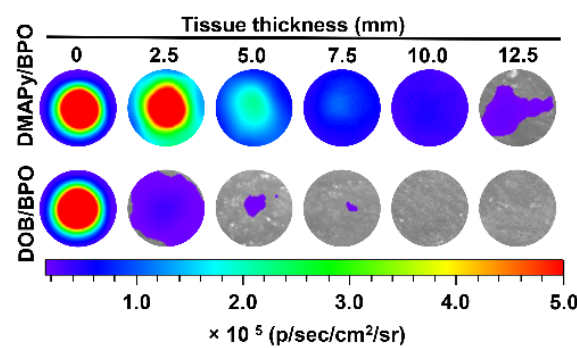

(h)

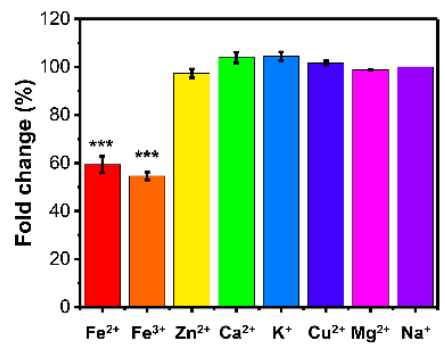

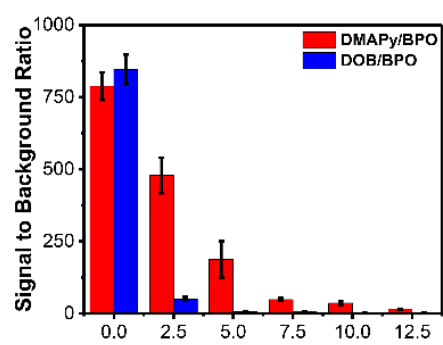

(i)

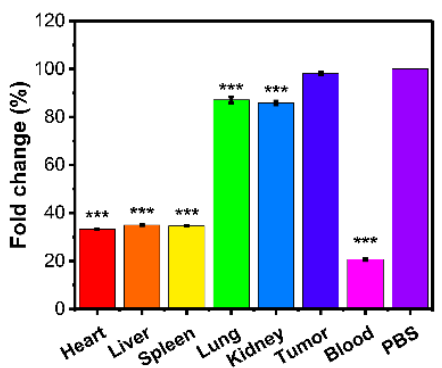

Fig. 5. | Phosphorescence properties of DMAPy/BPO and DOB/BPO nanoparticles. a, Diameter distribution of DMAPy/BPO nanoparticles. Inset: TEM image, scale bar $=100 \mathrm{~nm}$. $\mathbf{b}$, The normalized phosphorescence intensities of DMAPy/BPO nanoparticles as a function of the cycle number of UV light irradiation $(n=3)$. c, The phosphorescence intensities as a function of the concentration of DMAPy/BPO nanoparticles $(n=3)$. d, Phosphorescence images of DMAPy/BPO and DOB/BPO nanoparticles $\left(4 \mathrm{mg} \mathrm{mL}^{-1}\right)$ captured by different filters. e, Phosphorescence images of DMAPy/BPO and DOB/BPO nanoparticles $\left(10 \mathrm{mg} \mathrm{mL}^{-1}\right)$ covered with different thicknesses of chicken tissues. f, The SBR ratios for covering with different thicknesses of chicken tissues in (e). g. Cytotoxicities of DMAPy/BPO and DOB/BPO nanoparticles against $4 \mathrm{~T} 1$ cells. The $4 \mathrm{~T} 1$ cells were incubated with $\mathbf{D M A P y} / \mathbf{B P O}$ and DOB/BPO nanoparticles at different concentrations for $8 \mathrm{~h}(\mathrm{n}=4)$. $\mathbf{h}$. Fold change plot of phosphorescence intensities of DMAPy/BPO nanoparticles in various metal ions. Error bars: mean \pm standard deviation $(\mathrm{n}=3)$. Triple asterisks represent $p<0.01$ compared with $\mathrm{Na}^{+}$. i. Fold change plot of phosphorescence intensities of DMAPy/BPO nanoparticles in different tissue homogenates. Error bars: mean \pm standard deviation $(\mathrm{n}=3)$. Triple asterisks represent $p<0.01$ compared with PBS. 
The applications in intravital phosphorescence imaging were further investigated. The solutions of DMAPy/BPO NPs and DOB/BPO NPs were subcutaneously injected into Balb/c nude mice, followed by imaging with an IVIS instrument in bioluminescent mode after the $365 \mathrm{~nm}$ handheld UV lamp irradiating for $1 \mathrm{~min}$. And the images were captured at $\mathrm{t}=10 \mathrm{~s}$ after removal of light source. Notably, to ensure the biosafety of the UV irradiation procedure, the phosphorescence signals were activated by the handheld UV lamp with a $10 \mathrm{~mW} \mathrm{~cm}^{-2}$ power density, which is lower than the maximum power exposure allowed for skin irradiation $\left(18 \mathrm{~mW} \mathrm{~cm}^{-2}\right) .{ }^{[48]}$ For comparison, the signals derived from DMAPy/BPO NPs and DOB/BPO NPs were also evaluated under the fluorescence mode, simultaneously. As displayed in Fig. 6a, the subcutaneous phosphorescence imaging result in living mice reveals that both phosphorescent signals from DMAPy/BPO and DOB/BPO nanoparticles can be observed at $10 \mathrm{~s}$ after the end of excitation. The SBR of DMAPy/BPO NPs and DOB/BPO NPs subcutaneous phosphorescence imaging at $10 \mathrm{~s}$ are 160 and 75 (Fig. $6 \mathrm{~b}$ ), respectively. In contrast the fluorescence signals of DMAPy/BPO NPs and DOB/BPO NPs could hardly be distinguished from the tissue autofluorescence. It is noteworthy that, although the skin thickness of mice is just approximately $0.5 \mathrm{~mm}$, the short wavelength emissive DOB/BPO NPs exhibit a lower SBR than DMAPy/BPO NPs in subcutaneous phosphorescence imaging due to the tissue scattering of the skin. These results are in accordance with Fig. 5e and 5f, and it also demonstrate that the long wavelength emission of DMAPy/BPO can effectively decrease the tissue scattering to obtain the high quality phosphorescent bioimaging. The phosphorescence imaging of lymph node was further investigated because the lymph node labeling is clinically important in guiding tumor surgery. As shown in Fig. 5c and 5d, the phosphorescence signal of the axillary lymph node could be detected clearly (SBR $=$ 55) while the fluorescence signal is indistinguishable. Thus, the lymph node imaging confirms the effectiveness of DMAPy/BPO NPs for phosphorescence tissue imaging.

Precise identification of complicated diseases, like cancer diagnosis, calls for high-performance imaging. Encouraged by the good performance of lymph node imaging, we further evaluated the phosphorescence imaging ability in cancer diagnosis in vivo. To probe the feasibility of using the long wavelength RTP materials in cancer diagnosis in vivo, the armpit tumor bearing mice were set up by 4T1 breast cancer cells. The solution of DMAPy/BPO NPs was injected through tail vein into the living mice. At $6 \mathrm{~h}$ post-injection, the signals of DMAPy/BPO NPs were activated by UV light for $1 \mathrm{~min}$. Next, the phosphorescent images were captured at $10 \mathrm{~s}$ after removal of the UV lamp excitation by applying the IVIS instrument in bioluminescent mode. Similarly, the fluorescence imaging was recorded at the same time for 
comparison. As displayed in Fig. 6e, the phosphorescence signal occurs in armpit tumor clearly. Thanks to the NIR phosphorescence emission without the interference of the autofluorescence, the SBR for the phosphorescence guided armpit tumor imaging is as high as 43 (Fig. 6f). Then the mice bearing armpit tumor were sacrificed, and the main tissues were excised for ex vivo phosphorescence imaging. It is well known that nano materials prefer to be enriched in reticuloendothelial system (RES) organs. Interestingly, only liver displayed a low phosphorescence signals and it is almost no phosphorescence signals were observed in other main organs (Supplementary Fig. S19). And this result might be attributed to the phosphorescence signal was quenched by liver because of its abundant blood supply, which is correspond with the result in Fig. 5i. Further, the tissue with phosphorescence signals were collected and stained with H\&E (Supplementary Fig. S20), which verified that was armpit tumor. And the main organs were stained with $\mathrm{H} \& \mathrm{E}$ as well. Compared to the main organs of PBS pre-treatment living mice, the DMAPy/BPO NPs did not cause obvious damage to these organs (Supplementary Fig. S21). This work validated that the RTP materials (DMAPy/BPO NPs) with both long wavelength and long-lifetime properties could serve as a potent probe candidate for imaging guided diagnosis by avoiding the interference of autofluorescence and reducing the tissue scattering.

(a)

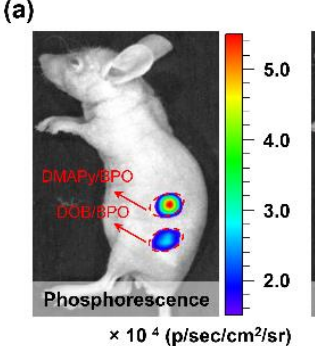

(c)

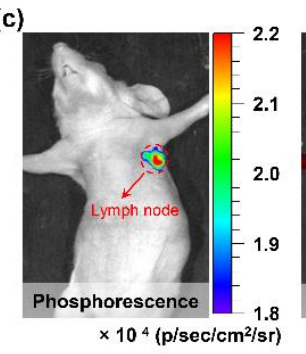

(e)

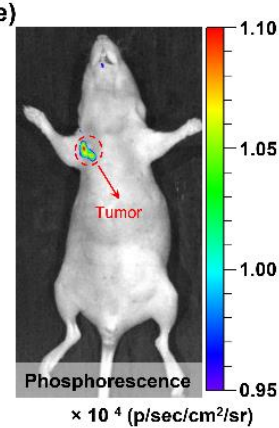

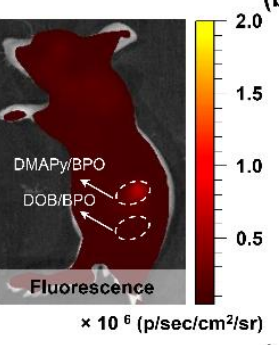

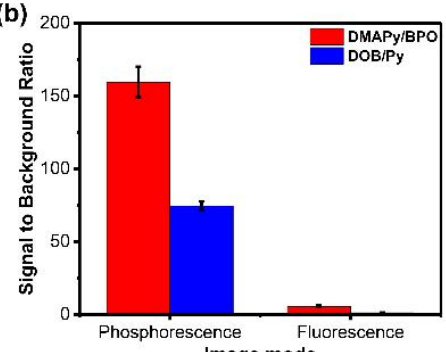

(d)

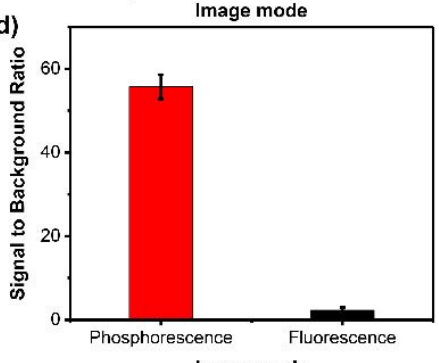

Image mode

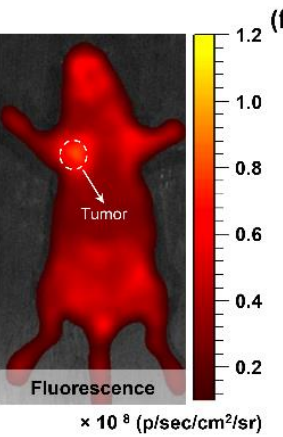

(f)

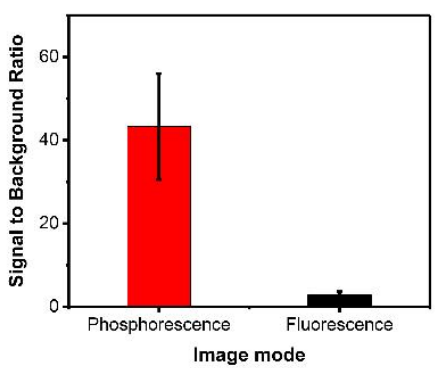


Fig. 6. The applications in intravital phosphorescence imaging. a, Phosphorescence and fluorescence imaging of a mouse with the subcutaneous inclusions of DMAPy/BPO NPs and DOB/BPO NPs (4 mg mL-1). The circles indicate the location of nanoparticle injection. $\mathbf{b}$, Signal to background ratio for phosphorescence and fluorescence imaging of subcutaneous injection in living mice. Error bars were based on standard deviation $(n=3)$. c, Phosphorescence and fluorescence imaging of lymph node in mice $0.5 \mathrm{~h}$ after the intradermal injection of DMAPy/BPO NPs (4 mg mL ${ }^{-1}$ ) into the forepaw of living mice. d, Signal to background ratio for phosphorescence and fluorescence imaging of lymph node in living mice. Error bars were based on standard deviation $(n=3)$. e, Phosphorescence and fluorescence imaging of a living tumor-bearing mice $6 \mathrm{~h}$ after the injection of DMAPy/BPO NPs into the vein $\left(4 \mathrm{mg} \mathrm{mL}^{-1}\right)$. f, Signal to background ratio for phosphorescence and fluorescence imaging of tumor in living mice. Error bars were based on standard deviation $(n=3)$.

\section{Conclusion}

A series of deep-red/near-infrared organic RTP materials with long lifetime are developed through one-axis two-wing guest-host strategy. The guest/pyrene derivatives with high conjugation are regarded as the central axis, which ensures that the materials have low $\mathrm{T}_{1}$ levels and long phosphorescence wavelengths. The host BPO plays a dual role of inhibiting the non-radiative transition of the guest excitons and promoting the intersystem crossing of the guest excitons. Through this strategy, the phosphorescence wavelength of the doped system can reach $732 \mathrm{~nm}$ and the phosphorescence lifetime reach $102 \mathrm{~ms}$. This is the first organic phosphorescent material with long wavelength (> $700 \mathrm{~nm}$ ) and long lifetime $(>100 \mathrm{~ms})$, and this material displayed a good tissue penetration. Furthermore, the potential of long wavelength RTP materials as a contrast agent candidate for high quality bioimaging was confirmed. Due to the elimination of tissue autofluorescence, the materials exhibit high-performance in both lymph node mapping and armpit tumor labeling with a high SBR of 55 and 43, respectively. This work provides a novel and feasible idea for solving the problem that low energy level excitons are prone to non-radiative transitions, and a practical solution for the construction of organic RTP materials with long wavelength and long lifetime.

\section{Methods.}

Sample preparation, theoretical calculation, details of photophysical measurements, NMR spectra and mass spectra. These materials are available free of charge online at http://......

\section{References}

1. Zhao, W., He, Z. \& Tang, B. Z. Room-temperature phosphorescence from organic 
aggregates. Nat. Rev. Mater. 5, 869-885 (2020).

2. Peng, Q. Ma, H. \& Shuai, Z. G. Theory of long-lived room-temperature phosphorescence in organic aggregates. Acc. Chem. Res. 54, 940-949 (2021).

3. Kabe, R., Adachi, C. Organic long persistent luminescence. Nature 550, 384-387 (2017).

4. Gu, L., Shi, H., Bian, L. et al. Colour-tunable ultra-long organic phosphorescence of a single-component molecular crystal. Nat. Photon. 13, 406-411 (2019).

5. An, Z., Zheng, C., Tao, Y. et al. Stabilizing triplet excited states for ultralong organic phosphorescence. Nat. Mater. 14, 685-690 (2015).

6. Wang, X., Shi, H., Ma, H. et al. Organic phosphors with bright triplet excitons for efficient X-ray-excited luminescence. Nat. Photon. 15, 187-192 (2021).

7. Bolton, O., Lee, K., Kim, H-J. et al. Activating efficient phosphorescence from purely organic materials by crystal design. Nat. Chem. 3, 205-210 (2011).

8. Wang, J., Gu, X., Ma, H. et al. A facile strategy for realizing room temperature phosphorescence and single molecule white light emission. Nat. Commun. 9, 2963 (2018).

9. Zhang, Y., Gao, L., Zheng, X. et al. Ultraviolet irradiation-responsive dynamic ultralong organic phosphorescence in polymeric systems. Nat. Commun. 12, 2297 (2021).

10. Bian, L., Shi, H. et al. Simultaneously enhancing efficiency and lifetime of ultralong organic phosphorescence materials by molecular self-assembly. J. Am. Chem. Soc. 140, 34, 10734-10739 (2018).

11. Ali Fateminia, S. M., Mao, Z. et al. Organic nanocrystals with bright red persistent room-temperature phosphorescence for biological applications. Angew. Chem. Int. Ed. 129, 12328-12332 (2017).

12. Dang, Q., Jiang, Y., Wang, J. et al. Room-temperature phosphorescence resonance energy transfer for construction of near-infrared afterglow imaging agents. Adv. Mater. 32, 2006752 (2020).

13. Wang, Y., Gao, H., Yang, J. et al. High performance of simple organic phosphorescence host-guest materials and their application in time-resolved bioimaging. Adv. Mater. 2007811 (2021).

14. Gao, H., Gao, Z. et al. Boosting room temperature phosphorescence performance by alkyl modification for intravital orthotopic lung tumor imaging. Small 17, 2005449 (2021).

15. He, Z., Gao, H. et al. Achieving persistent, efficient, and robust room-temperature phosphorescence from pure organics for versatile applications. Adv. Mater. 31, 1807222 (2019).

16. Zhang, T., Ma, X., Wu, H. et al. Molecular engineering for metal-free amorphous 
materials with room-temperature phosphorescence. Angew. Chem. Int. Ed. 59, 1120611216 (2020).

17. Li, J., Zhou, J., Mao, Z. et al. Transientand persistent room-temperature mechanoluminescence from a white-light-emitting AIEgen with tricolor emission switching triggered by light. Angew. Chem. Int. Ed. 130, 6559 -6563 (2018).

18. Wang, T., Hu, Z., Nie, X. et al. Thermochromic aggregation-induced dual phosphorescence via temperature-dependent $s p^{3}$-linked donor-acceptor electronic coupling. Nat. Commun. 12, 1364 (2021).

19. Yang, Z., Xu, C., Li, W., Mao, Z. et al. Boosting the quantum efficiency of ultralong organic phosphorescence up to $52 \%$ via intramolecular halogen bonding. Angew. Chem. Int. Ed. 59, 17451-17455 (2020).

20. Zhang, Y., Gao, L., Zheng, X. et al. Ultraviolet irradiation-responsive dynamic ultralong organic phosphorescence in polymeric systems. Nat, Commun, 12, 2297 (2021).

21. Dou, X., Zhu, T., Wang, Z. et al. Color-tunable, excitation-dependent, and time-dependent afterglows from pure organic amorphous polymers. Adv. Mater. 32, 2004768 (2020).

22. Ren, J., Wang, Y. et al. Force-induced turn-on persistent room-temperature phosphorescence in purely organic luminogen. Angew. Chem. Int. Ed. 60, 1-7 (2021).

23. Li, D., Lu, F., Wang, J. et al. Amorphous metal-free room-temperature phosphorescent small molecules with multicolor photoluminescence via a host-guest and dual-emission strategy. J. Am. Chem. Soc. 140, 1916-1923 (2018).

24. Zhou, B. \& Yan, D. P. Hydrogen-bonded two-component ionic crystals showing enhanced long-lived room-temperature phosphorescence via TADF-assisted förster resonance energy transfer. Adv. Funct. Mater. 29, 1807599 (2019).

25. Nidhankar, A., Goudappagouda. et al. Self-assembled helical arrays for the stabilization of the triplet state. Angew. Chem. Int. Ed. 59,13079-13085 (2020).

26. Cai, S., Ma, H., Shi, H. et al. Enabling long-lived organic room temperature phosphorescence in polymers by subunit interlocking. Nat. Commun. 10, 4247 (2019). 27. Xiao, F., Wang, M., Lei, Y. et al. Achieving crystal-induced room temperature phosphorescence and reversible photochromic properties by strong intermolecular interactions. J. Mater. Chem. C 8, 17410-17416 (2020).

28. Xiao, L., Wu, Y., Yu, Z. et al. Room-temperature phosphorescence in pure organic materials: halogen bonding switching effects. Chem. Eur. J. 24, $1801-1805$ (2018).

29. Wu, X., Huang, C-Y., Chen, D-G. et al. Exploiting racemism enhanced organic room-temperature phosphorescence to demonstrate Wallach's rule in the lighting chiral chromophores. Nat. Commun. 11, 2145 (2020).

30. Wang, X., Guo, W., Xiao, H. et al. Pure organic room temperature 
phosphorescence from unique micelle-assisted assembly of nanocrystals in water. Adv. Funct. Mater. 30, 1907282 (2020).

31. Wang, X., Xiao, H., Chen, P. et al. Pure Organic room temperature phosphorescence from excited dimers in self-assembled nanoparticles under visible and near-infrared irradiation in water. J. Am. Chem. Soc. 141, 5045-5050 (2019).

32. Ono, T., Kimura, K., Ihara, M. et al. Room-temperature phosphorescence emitters exhibiting red to near-infrared emission derived from intermolecular charge-transfer triplet states of naphthalenediimide-halobenzoate triad molecules. Chem. Eur. J. DOI: 10.1002/chem.202100906.

33. Katsurada, Y., Hirata, S., Totani, K. et al. Photoreversible on-off recording of persistent room-temperature phosphorescence. Adv. Opt. Mater. 3, 1726-1737 (2015). 34. Zhang, X., Du, L., Zhao, W. et al. Ultralong UV/mechano-excited room temperature phosphorescence from purely organic cluster excitons. Nat. Commun. 10, 5161 (2019).

35. Chen, C., Chi, Z., Chong, K. C. et al. Carbazole isomers induce ultralong organic phosphorescence. Nat. Mater. 20, 175-180 (2021).

36. Alam, P., Leung, N. C., Liu, J. K. et al. Two are better than one: a design principle for ultralong-persistent luminescence of pure organics. Adv. Mater. 32, 2001026 (2020).

37. Chen, Y., Xie, Y., Shen, H., Lei, Y. et al. Tunable phosphorescence/fluorescence dual emissions of organic isoquinoline-benzophenone doped systems by akoxy engineering. Chem. Eur. J. 26, 17376-17380 (2020).

38. Chen, B., Huang, W., Su, H. et al. An unexpected chromophore-solvent reaction leads to

bicomponent aggregation-induced phosphorescence. Angew. Chem. Int. Ed. 59, 10023-10026 (2020).

39. Wang, Y., Yang, Jie., Fang, Manman. et al. Forster resonance energy transfer: An efficient way to develop stimulus-responsive room-temperature phosphorescence materials and their applications. Matter 3, 449-463 (2020).

40. Lei, Y., Dai, W., Tian, Y. et al. Revealing insight into long-lived room-temperature phosphorescence of host-guest systems. J. Phys. Chem. Lett. 10, 6019-6025 (2019).

41. Lei, Y., Yang, J., Dai, W. et al. Efficient and organic host-guest room-temperature phosphorescence: tunable triplet-singlet crossing and theoretical calculations for molecular packing. Chem. Sci. 12, 6518-6525 (2021).

42. Wang, D., Xie, Y., Wu, X. et al. Excitation-dependent triplet-singlet intensity from organic host-guest materials: tunable color, white-light emission, and room-temperature phosphorescence. J. Phys. Chem. Lett. 12, 1814-1821 (2021).

43. Lei, Y., Dai, W., Guan, J. et al. Wide-range color-tunable organic phosphorescence 
materials for printable and writable security inks. Angew. Chem. Int. Ed. 59, 1605416060 (2020).

44. Wei, J., Liang, B., Duan, R. et al. Induction of strong long-lived room-temperature phosphorescence of $\mathrm{N}$-phenyl-2-naphthylamine molecules by confinement in a crystalline dibromobiphenyl matrix. Angew. Chem. Int. Ed. 128, 15818-15822 (2016). 45. Xie, Z., Zhang, X. et al. Wide-range lifetime-tunable and responsive ultralong organic phosphorescent multi-host/guest system. Nat. Commun. 12, 3522 (2021).

46. Wang, X., Sun, Y., Wang, G., Li, J., Li, X., Zhang, K. TADF-type organic afterglow. Angew. Chem. Int. Ed. DOI: 10.1002/anie.202105628.

47. Chen, B., Huang, W., Nie, X., Cheng, X., Liao, F., Miao, H., Zhang, X., Zhang, G. Organic guest-host system produces room-temperature phosphorescence at part-per-billion level. Angew. Chem. Int. Ed. DOI: 10.1002/anie.202106204.

48. Zhen, X., Tao, Y., An, Z., Chen, P., Xu, C., Chen, R., Huang, W., Pu, K. Adv. Mater, 29, 1606665 (2017).

49. Dang, Q., Jiang, Y., Wang, J., Wang, J., Zhang, Q., Zhang, M., Luo, S., Xie, Y., Pu, K., Li, Q., Li, Z. Room-temperature phosphorescence resonance energy transfer for construction of near-infrared afterglow imaging agents. Adv. Mater. 32, 2006752 (2020).

50. Liu, X., Dai, W., Qian, J., Lei, Y., Liu, M., Cai, Z., Huang, X., Wu, H., Dong, Y. Pure room temperature phosphorescence emission of an organic host-guest doped system with a quantum efficiency of 64\%. J. Mater. Chem. C, 9, 3391 (2021).

51. Wang, Y., Gao, H., Yang, J., Fang, M., Ding, D., Tang, B. Z., Li, Z. High performance of simple organic phosphorescence host-guest materials and their application in time-resolved bioimaging. Adv. Mater. 33, 2007811 (2021).

\section{Acknowledgements}

This work is supported by the National Natural Science Foundation of China (No 22071184) and the Zhejiang Provincial Natural Science Foundation of China (No LY20B020014).

\section{Author contributions}

Y. L, X. H. and D. D. designed the research work and revised the manuscipt. F. X. synthesized the materials. F. X. and Y. L. carried out photophysical property measurements. H. G. carried out biological tissue measurements. X. Z. carried out density functional theory calculations. Y. L, X. H. and D. D. wrote the manuscript. M. L, Z. C. and H. W. edited the manuscript. All authors discussed the results and commented on the manuscript. 
